\title{
INCONSISTENCIA DE LA DIMENSIÓN ANALÍTICA-EMPÍRICA \\ DESDE LA CONFORMACIÓN CEREBRAL COGNITIVA
}

Inconsistency of the analytic-empirical

dimension from cognitive brain conformation

\author{
RÓMUlo IGNACIO SAN MARTín GARCía* \\ Universidad Politécnica Salesiana/Quito-Ecuador \\ rsanmartin@ups.edu.ec
}

\begin{abstract}
Resumen
Hay una inconsistencia de la perspectiva analítica filosófica de frente a la estructura cerebral; ésta es más que regresión hacia los átomos del conocimiento, que para los analíticos clásicos está en el exterior.

A la arquitectura cognitiva cerebral se la puede vislumbrar como un todo sintético que conjunta las dimensiones internas de la naturaleza humana con la externa, es decir que toma en cuenta tanto la exterioridad como la naturalidad y parte de ambas; en efecto el ordenamiento inteligente sabe bordar lo instintivo y límbico con los datos que le da la sensorialidad y a su vez lo instintivo-límbico en lo lingüístico-lógico y lo sensorial en lo lingüístico-lógico para la planificación y la decisión, elementos muy propios y al mismo tiempo simultáneos en la estructura cerebral. Por lo tanto, el absolutizar la analítica es realmente una reducción del conocimiento, más aún, la reducción del significado de los contenidos a los hechos y a la materia, es un contradictorio cerebral, por la naturaleza misma de su estructura que no parcializa ni reduce nada como si es el caso de lo analítico.

El empirismo clásico y el del siglo pasado delante de los estudios de la estructura y funcionamiento del cerebro, resulta ser parcial, pues poseen una estructura reduccionista que se asimila totalitaria. No es que sea inválido totalmente, sino que es parcialmente válido, es decir que, si se analizan los contenidos desde la sensorialidad cerebral, es más o menos coherente, pero actualmente, quedarse sólo en ese campo e interpretación, e impertinencia cognitiva.
\end{abstract}

Palabras clave

Inteligencia cristalizada, inteligencia fluida, sistema límbico, empirismo, analítico.

Forma sugerida de citar: San Martín García, Rómulo Ignacio (2017). Inconsistencia de la dimensión analíticaempírica desde la conformación cerebral cognitiva. Sophia, colección de Filosofía de la Educación, 22(1), pp. 55-81.

* Ph.D. en Filosofía. Docente investigador en cerebro y ciencias cognitivas. Director de la Carrera de Filosofía y Pedagogía de la Universidad Politécnica Salesiana.

Código Orcid: orcid.org/0000-0002-7414-4598 


\begin{abstract}
There is an inconsistency of the philosophical analytic perspective in front of the brain structure; this is more than regression towards the atoms of knowledge, which for the classical analytic is on the outside.

The cerebral cognitive architecture can be seen as a synthetic whole that combines the internal dimensions of human nature with the external, that is to say that takes into account both the exteriority and the naturalness and part of both; The intelligent ordering knows how to embroider the instinctive and limbic with the data that gives the sensoriality and in turn the instinctivelimbic in the linguistic-logical and the sensorial in the linguistic-logic for the planning and the decision, very own elements and Simultaneously simultaneous in the brain structure. Therefore, to absolutize the analytic is really a reduction of knowledge, moreover, the reduction of the meaning of content to facts and matter, is a contradictory brain, by the very nature of its structure that does not partialize or reduce anything As if it is the case of the analytic.

The classic empiricism and that of the last century in front of the studies of the structure and functioning of the brain, turns out to be partial, because they possess a reductionist structure that is assimilated totalitarian. It is not that it is totally invalid, but it is partially valid, that is to say, if the contents are analyzed from the cerebral sensoriality, it is more or less coherent, but at present, to remain only in that field and interpretation, and cognitive impertinence.
\end{abstract}

Keywords

Crystallized intelligence, Fluid intelligence, limbic system, empiricism; analytical.

\title{
Introducción
}

El estudio de la filosofía analítica ha marcado el ritmo del pensar filosófico en el s. XX. Al mismo tiempo que se investigaba y reflexionaba en torno a las ciencias físicas y químicas-biológicas, que tienen un método analítico-sintético, se consolidaba también la ciencia humana con un método analítico cautivador. Esta tendencia por fin entró en el mundo computacional, el cual se articuló prestísimo con el mundo cognitivo (computacional-cognitivo), causando la analiticidad del conocimiento en el campo tecnológico. Con estos eventos, era normal que se identificase que, todo lo que es ciencia es rigurosa, si es analítica.

Contemporáneamente viene el estudio del cerebro de modo científico y se asocia los términos cerebro-mente. No es una cuestión de cerebro-orden, que se vincula a la sintáctica, sino de elementos que no tienen su referente sólo en la exterioridad, sino que están insertados en la naturaleza complejísima del cerebro. Entonces el cerebro-mente acaba con la visión sintáctica de la ciencia y por tanto de la filosofía, puesto que ésta está relacionada con los eventos mentales.

Los estudios e investigaciones cerebrales ponen en tela de juicio la sola sintaxis y abre una vía para la semántica. El cerebro no es sólo sensorial, evento al cual se asocia inmediatamente la dimensión analítica, 
sino que tiene otras áreas biológicas que articulan los datos y les ponen vitalidad y causalidad. Así que el estudio científico del cerebro pone en crisis la dimensión computacional (analítica) cognitiva y se asume una versión más analítica-sintética.

El objetivo es demostrar que el cerebro es más que analítico y la visión empirista referencialista e ideacionalista moderna y contemporánea es errónea, porque se centró en el funcionamiento de un área cerebral, a la cual sacrificó otras perspectivas del conocer, sobre todo de la dimensión biológica.

La hipótesis que guía este problema, en este artículo, es que la estructura cerebral no es sólo analítica, sino que, porque el cerebro humano es instintivo, límbico, sensorial, lógico, planificador y pragmático, es analítico y sintético-vital.

El método que se usa en este estudio es bibliográfico y hermenéutico, en efecto desde la indagación de la estructura cerebral se puede constatar que el área sensorial, más analítica, es sólo una parte del diseño cerebral y que las otras dimensiones son más vitales-sintéticas-volitivas. Sobre esos datos, la tarea es reflejar la composición analítica y descubrir que el cerebro es más que analítico.

La proyección del estudio es amplia. En la medida que se conozca más la estructura y funcionamiento de las áreas cerebrales se tendrá más luces para interpretar mejor la naturaleza del conocimiento más allá de la dimensión analítica y sintética, sino por fin ya de modo causal y por fin hasta analógico. Así se procede a comprender mejor el cerebro intuitivo y cognitivo.

\section{El cerebro instintivo y cognitivo}

Cada uno tiene su estructura funcionan. El instintivo reacciona inmediatamente y es intrínseco al sujeto. El afectivo es dependiente del externo y el cognitivo es una construcción externa e interna y no es inmediato.

El cerebro instintivo tiene más experiencia del pasado; el ambiente no está todo ordenado y definido, antes bien es desafiante y preparado para reaccionar a los datos imprevistos; por ejemplo, si para reaccionar inmediatamente, antes una situación de peligro, tuviésemos que mediar por la dimensión cognitiva, el ser humano sería víctima de la calamidad y sería fatal. En muchos eventos el humano necesita reaccionar instintivamente, sobretodo de frente a los peligros tales como los riesgos de daños, el escape, el miedo (Olsson, 2016, p. 8). En este sentido la mediación cognitiva es más lenta, puesto que tiene que procesar más datos, de modo que, si dejásemos que mediase la dimensión cognitiva, el ser humano 
fracasaría. El cerebro cognitivo tiene un retraso de la mitad con respecto al instintivo. Llega tarde cuando está mediando las cosas demasiado. La presencia del signo cognitivamente llega 0,5 segundos después que se estuvo atento al signo que lo generó. Pinker, con parte de razón, pero de modo absoluto, sostiene que los animales se conducen por instinto, en desventaja a los humanos que se comportan racionalmente (Pinker, 2010); cierto que el humano es racional, pero la dimensión instintiva es parte de nuestra naturaleza, por ello es peligroso paralelizar el instinto para los animales y la racionalidad para los humanos.

Es bueno hacer reaccionar en sorpresa ante las eventualidades, sobretodo en planes de contingencia delante de catástrofes. En el campo pedagógico conviene la presentación de pruebas sin previo aviso o de actividades sin predisposición para que justamente se ejerciten los conocimientos ante la calamidad, que se complementa con la cognición relacionado con lo social.

\section{Cognición relacionada con lo social}

El sistema límbico de la parte posterior del orbitofrontal cortex, el área 13 , es el responsable de las reacciones emocionales a los estímulos sociales, está presente en 13 humanoides y también en el humano y, éste, tiene este córtex más pequeño que el orangután, el cual tiene más expandido, por ello quizás menos social y menos emotivo (Semendeferi, Schleicher, Zilles, \& Hoesen, 1998). El área frontal regula la social cognition, por ello se puede comparar tanto el Síndrome de William con el autismo, el primero está en relación a una cognición social hiperafiliativa, busca estar en exagerado reconocimiento social, en cambio el segundo es la evasión de esa. Tanto en la una como en la otra hay una alteración de la densidad neuronal, sobre todo en las áreas de broadman.

El cerebro cognitivo está relacionado con la inteligencia y la racionalidad. Inteligencia es "la habilidad para razonar, planear, solucionar problemas, pensar abstractamente, comprender ideas complejas, aprender rápidamente y aprender de la experiencia" (Gottfredson, 1997, p. 13). Si se lo pone en secuencia, los actos tendrían esta secuencia.

- Aprender de la experiencia

- Aprender rápidamente

- Comprender ideas complejas

- Habilidad para razonar

- Pensar abstractamente

- Planear y solucionar problemas 
Por motivos fundamentales relacionados con el pensar analítico, vale la pena concentrase solamente en el aprender de la experiencia y el aprendizaje rápido.

El primer acto es tener experiencia, para lo cual están los sentidos relacionados con el evento externo y con el área límbica. Las repeticiones constantes de los estímulos ocasionan que se fragüe la inteligencia, por lo que, de la repetición, el sujeto se percata que puede aplicarles a otras realidades, las cuales no estuvieron incluidas en los ejercicios iniciales, para así sacar experiencia, que puede ser aplicada a otros campos. Tal es el caso de la evasión, o el caso del peligro, o del ciego, o de la experiencia amorosa, con similares características se aplica al nuevo espacio, por el cual sabe si le conviene o no. Si se aplica esto a la enseñanza-aprendizaje, pues es la pragmatización de un aprendizaje para otra realidad tal como la aplicación de un experimento en otros laboratorios y campos, con el fin de ver la reacción. Pensemos por ejemplo la aplicación del gusto por la música, que eleva a los adolescentes a la experiencia religiosa.

En segunda instancia tenemos el aprender. Esto es encender, para el cual se necesita la base y a eso el elemento nuevo, que encienda. Los datos no son del sujeto, pero tienen el combustible que sostendrá el encendimiento. Así el aprender es la alianza neuronal delante de los datos presentados, que comienzan a relacionarlos creando redes para posteriores aprendizajes. Si muchas velas apagadas no encienden una, entonces basta una para encender las muchas. Esto significa que el aprender es la entrada para la aplicación encendida en nuevos campos. Ello es la secuencia de los aprendizajes. La experiencia aplicada a otros campos crea destrezas en el sujeto, tiene la ligera certeza que las cosas están en relación y que los aprendizajes no son cerrados, sino con ventanas, por las cuales la información es relativa a otros campos. Esto es la destreza. Así el aprender es la asimilación de la información primero para el campo deseado y posteriormente su utilización a otros campos. Se aprende más rápido cuando se pone en relación, por lo que el criterio de relación permite que fluya los conocimientos.

El aprender inicial es complicado y requiere mucho ambiente enriquecido, pues no hay ni la memoria. En el cerebro están las neuronas muy separadas y se irán enredando en la medida que sean estimuladas sensorialmente; así que la independencia primigenia se acaba al ser estimulada por eventos ajenos a sí. Esta es la causa, la independencia y separación de las neuronas, para que los aprendizajes tempranos sean lentos, puesto que el encendido que experimentan las neuronas comporta la inserción de datos, de manera que la información quede en las redes neuronales. Este contexto es el fundamento para que el ambiente sea enriquecido con grandes even- 
tos que causen reacción a las neuronales. Un ambiente pobre, al menos si no se tiene experiencia, no permite el desarrollo de las redes neuronales. La mente en los primeros años es similar a como una sábana en blanco o como un libro de notas vacío, las cuales se van primigeniamente llenando bajo la modalidad de premio y castigo y reforzamiento (Turing, 1950). La mente del niño, entonces está para ser estimulada en los primeros momentos, para los cuales requiere de un ambiente enriquecido, por lo cual sería algo semejante a una máquina inteligente que se le va incorporando la información (Turing, 1950). Las neuronas, de frente a los datos, se relacionan y dan lugar a la memoria, la cual es el archivo de los datos, que posteriormente relativizan el exterior y tienen, el dato, de modo digital; ella exime la presencia del estímulo inicial, pues tiene ya la memoria. Así que cuando desarrolla esa, usa esos datos para nuevo aprendizaje: si en un inicio se necesitó el cuerpo y el estímulo exterior para formar las redes, en la medida que crece el árbolred se usan esos datos para aprendizajes nuevos. El aprender se hace digital en la medida que se usan los datos de los datos.

El ambiente externo, con sus cuerpos, en relación con las áreas cerebrales sensoriales da lugar a las sensaciones, de las cuales aparecerán, por síntesis sensorial las ideas, que son una red que combina datos neuronales del mundo interior instintivo límbico con los datos del exterior.

Para vislumbrar de mejor forma el desarrollo de la idea antes expuesta resulta importante analizar la naturaleza no computacional de la inteligencia.

\section{La naturaleza no computacional de la inteligencia}

La inteligencia, como estructura humana para el aprendizaje, no es solo un almacén de los datos que exteriormente existen, los cuales hasta sensorialmente se los puede separar, sino que, en cuanto facultad, articula esos datos externos con los recursos propios que le han hecho una facultad. Los datos al insertarse en el humano no quedan similares al exterior, sino que se abstraen. Éste proceso es inteligente, es decir que los datos son "leídos" por la facultad, similar a un escáner, que además fotocopia el dato, lo articula creativamente, para otros fines y objetivos. En este sentido la inteligencia no es una especulación del dato, sino una transformación creativa, a través de las categorías, para otros fines.

Bajo este horizonte se puede reflexionar que el área cerebral, en donde se estructura la inteligencia como facultad, no es receptora y archivo de datos, sino una estructura lógica que sintetiza lo recibido con los 
datos propios de sí. El fondo de esto es la flexibilidad cerebral. El dato por sí mismo no es flexible, sino que es la estructura la que organiza, solapa y transforma el dato. Así que la modalidad no viene por el dato, sino por la facultad inteligente. Igualmente, desde aquí se entiende mejor el aprendizaje relacionado con el cerebro y la vida social, en el cual el lenguaje es la esencia más alta para aprender, hasta culturalmente. Por otro lado, desde este horizonte se comprende la construcción de máquinas inteligentes fundadas en la estructura del cerebro. Con todo lo anterior, se tornará difícil sostener una visión analítica pura del lenguaje y del pensamiento.

Analizamos por ello estas fases: la estructura cognitiva flexible, la inteligencia no computacional, la dimensión social cultural y lingüística del cerebro, teniendo en cuenta como un primer momento de análisis la estructura.

\section{La estructura cognitiva flexible}

La estructura cognitiva es variada, tiene áreas específicas con funcionalidad propia; el cerebro no es un factotum desde las mismas estructuras, sino es diversificado. Tiene estructuras sensoriales, motoras, lingüísticas, de planificación y decisión superior. Es un cerebro ordenado. Esta naturaleza podría despertar un sentido de ser un cerebro modular inflexible, por tanto, de estructuras cognitivas independientes y autosuficientes. Esta perspectiva parece proyectar la versión de la modularidad de Fodor (1983), es decir que la mente es un conjunto de esquemas medio inflexibles. Sin embargo, el cerebro es flexible; las áreas cerebrales y los módulos específicos son interrelacionados entre ellos para tener productos de gran alcance.

Este evento es producto de la evolución de la cognición en sus facultades, así que no evoluciona como un todo sino por la parte en función del todo, lo cual favorece a todo el sistema, puesto que, si se dañase un área, no por ello queda perjudicado el sistema, sino que en su flexibilidad entran en acción otros campos. La evolución trabaja de ese modo en la formación del todo desde la parte, que la va tallando paulatinamente, así que es adaptable puesto que los impulsos no son genéricos, sino específicos, ello hace el mismo órgano similar en los sujetos tenga diferencia, no funcional, pero sí de intensidad en el funcionamiento de esa área.

La traducción pedagógica en la educación del sujeto, indica la estimulación cognitiva de un área ya formada, pero que debe ser usada para aprender en el espacio actual, o del aprendizaje de un estímulo preciso. $\mathrm{Si}$ hubo adaptación del órgano en la conformación biológica, entonces debe haberla en el aprendizaje. Pensemos el caso de la enseñanza a los sordos 
mudos que está atrofiada el área temporal y la Brocca, pero no por ello está hipotecado el entendimiento, por eso resulta necesario crear didácticas que impulsen otros órganos para el aprender. Si fuese todo modular inflexibles, entonces, no pudiese aprender, pues cae en una versión computacional de la mente, en el cual se atrofia el sistema.

Para comprender de mejor forma la estructura cognitiva flexible y de forma general la naturaleza computacional, analizamos a continuación la inteligencia no computacional, donde se presentará un análisis en torno al cuadro construido desde los estudios de (Cattell-Horn-Carroll theory of cognitive abilities-CHC theory).

\section{La inteligencia no computacional}

La inteligencia, o sea la capacidad de leer internamente, en contraposición a los sentidos que leen el externo, tiene diversidad de manifestaciones. Esta se puede codificar en dos tareas como efecto: la cristalización y la fluidez.

En cuanto a la cristalización. La inteligencia es capaz de usar destrezas conocimientos, experiencias, que están en la memoria (Judith M. Burkart M. N., 2016); estos conocimientos son pesados y logran cristalizarse en otros aprendizajes, es decir que son contenidos modelos que son aplicables a otros campos; es similar a como enviar el contenido por diversos canales, pero siempre manteniéndose el mismo contenido.

Este acto se aplica en la utilización del mismo material en técnicas didácticas para la asunción del contenido, es decir que el mismo contenido puede ser tomado bajo diversas técnicas, con el fin didáctico; ello es para cristalizar el saber.

En cuanto a la inteligencia que fluye, es la capacidad de pensar lógicamente y resolver problemas en nuevas situaciones independientemente del conocimiento adquirido previamente. Ello implica que es un conocimiento de relación. El uso del material no es real, sino por relación lógica, en el cual tiene cabida la abstracción. Esta es la separación de los datos para presentar nuevos eventos; es similar a la transformación de la materia en energía, o el agua en estado líquido que pasa a estado sólido o en vapor. Este conocimiento es el más rico porque no gobiernan los contenidos, sino la estructura digital, es decir de abstraer a términos más generales.

La inteligencia incluye varios ámbitos; su característica es la articulación de las dimensiones que están separadas y que se explican a sí mismas, tales como la instintiva, la límbica, la sensorial. Entonces ser inteligente no es una condición exclusivamente computacional, sino la capacidad de tejer las áreas cerebrales y los cerebros (antiguo, medio y neo) en una visión de 
conjunto. En este sentido la inteligencia teje y pone en teleología el instinto, lo mismo que la dimensión límbica, como también los sentidos. Además, inteligencia es la capacidad de combinar los contenidos interiores con los exteriores; en efecto lo que está separado, la una. En este sentido inteligencia es la capacidad leer interiormente. La facultad inteligente humana es evolución, por ello hay que compararla con la de los animales. Si el comportamiento no está bordado de inteligencia pues es sólo comportamiento, pero si se articula más contenido aprendido y sintético, se hace comportamiento inteligente. Los humanos tenemos comportamiento inteligente, por medio del cual se asocia las facultades cognitivas a los miembros del cuerpo (manos, piernas, brazos), sin por ello reducirse a eso. Un comportamiento inteligente, si bien será mecánico, pero para llegar a aquel tuvo que practicar y ejercitarse, no es programado, sino flexible. Ésta se encuentra asociada a cierta facultad mental (Tomasello \& Call, 1997).

La inteligencia por tanto forma una totalidad desde la unión intencional de los diversos elementos. En el siguiente cuadro construido desde los estudios de (Cattell-Horn-Carroll theory of cognitive abilities$\mathrm{CHC}$ theory) presenta la estructura de la inteligencia en su complejidad.

\section{Cuadro 1}

Estructura de la inteligencia en su complejidad

\begin{tabular}{|c|l|}
\hline Habilidad & \multicolumn{1}{c|}{ Descripción } \\
\hline $\begin{array}{c}\text { Razón lógica } \\
\text { (razonamiento } \\
\text { fluido) }\end{array}$ & $\begin{array}{l}\text { Va a la solución de nuevos problemas, por ello requiere: elemen- } \\
\text { tos inductivos: formación y clasificación de conceptos, identi- } \\
\text { ficación de relaciones, comprensión de implicaciones; de aquí } \\
\text { deductivamente: diseño de inferencias, solución de problemas, } \\
\text { extrapolación, transformación de la información. } \\
\text { Producto: razonar inductiva y deductivamente, rapidez en el } \\
\text { razonamiento. }\end{array}$ \\
\hline $\begin{array}{l}\text { Conocimiento } \\
\text { comprensivo } \\
\text { (inteligencia } \\
\text { cristalizada) }\end{array}$ & $\begin{array}{l}\text { Es proceso de incorporación de la información: mira la in- } \\
\text { formación y la manera como la inserta y la profundiza a través } \\
\text { terno. Es un conocimiento del "qué", o sea a través de la lengua, } \\
\text { porayecto inserta otras informaciones y habilidades y hasta expe- } \\
\text { riencias de la vida. } \\
\text { Implica habilidades lingüísticas: desarrollo lingüístico, en el que } \\
\text { está el aumento del léxico; capacidad de escuchar (superación } \\
\text { del ojo), información verbal, información de la cultura y del } \\
\text { entorno; del escuchar desarrolla la comunicación: el diálogo, } \\
\text { que le da fluidez verbal, desarrollo de la gramática, interés por }\end{array}$ \\
el desarrollo de una lengua extranjera
\end{tabular}




\begin{tabular}{|c|c|}
\hline Habilidad & Descripción \\
\hline $\begin{array}{l}\text { Inteligencia } \\
\text { vinculada a } \\
\text { memoria a corto } \\
\text { término }\end{array}$ & $\begin{array}{l}\text { Habilidad de retención de información inmediata, en pocos } \\
\text { minutos antes y rápidamente se desvanece. } \\
\text { Habilidad: Memoria spam, y memoria de trabajo, que tiene una } \\
\text { visión general, sin diferenciar ni analizar. }\end{array}$ \\
\hline $\begin{array}{l}\text { Inteligencia } \\
\text { visual }\end{array}$ & $\begin{array}{l}\text { Habilidad para generar, almacenar y recuperar información } \\
\text { sensitiva visual. Ello implica que el sujeto pueda percibir y } \\
\text { transformar las formas y operaciones visuales que se mantie- } \\
\text { nen y cambian en el espacio, por ello desarrolla el sentido de } \\
\text { orientación. } \\
\text { Desarrolla el sentido de relación visión con espacio, velocidad } \\
\text { de cierre, memoria visual, escaneamiento espacial, integración } \\
\text { serial (sentido de continuidad), estimación amplia, ilusión per- } \\
\text { ceptual, imagen. }\end{array}$ \\
\hline $\begin{array}{l}\text { Inteligencia } \\
\text { auditiva }\end{array}$ & $\begin{array}{l}\text { Habilidad de diferenciar el sonido en medio del ruido, a tra- } \\
\text { vés del oído. Descubre un significado en medio del ruido. Por } \\
\text { ello organiza y discrimina los sonidos. Le abre a la capacidad } \\
\text { de analizar los sonidos por los cuales podrá sintetizar nuevas } \\
\text { soluciones auditivas y grupos de sonidos. } \\
\text { La habilidad: Codificación fonética, diferenciación de sonidos } \\
\text { discursivos, resistencia a la distorsión de sonidos auditivos, me- } \\
\text { moria para modelos de sonido, apertura a la huella temporal } \\
\text { que desemboca en la medición del tiempo mediante el ritmo, } \\
\text { asociación discriminación intensidad y duración, frecuencia } \\
\text { del sonido (melodía), localización del sonido, escuchar, y factor } \\
\text { umbral de la conversación. }\end{array}$ \\
\hline $\begin{array}{l}\text { Inteligencia con } \\
\text { memoria a largo } \\
\text { plazo (capacidad } \\
\text { de recuperar) }\end{array}$ & $\begin{array}{l}\text { Habilidad para almacenar la información por largo plazo (en- } \\
\text { tendido más de una hora) y recuperar lo guardado (ideas, con- } \\
\text { ceptos) por vía asociativa. Esta capacidad permite fluidez. } \\
\text { Habilidades: memoria asociativa, memoria significativa, me- } \\
\text { moria de llamamiento libre, ideacional fluidez, fluidez asociati- } \\
\text { va, expresión y nominación fluida, sensibilidad a los problemas, } \\
\text { capacidad de aprender. }\end{array}$ \\
\hline $\begin{array}{c}\text { Inteligencia del } \\
\text { proceso cognitivo }\end{array}$ & $\begin{array}{l}\text { Habilidad para, automáticamente o con fluidez, preformar } \\
\text { operaciones cognitivas elementales, para lo cual se necesita } \\
\text { concentración. } \\
\text { Habilidad: velocidad perceptual, facilidad numérica, velocidad } \\
\text { de razonamiento, velocidad de lectura y velocidad de escritura. }\end{array}$ \\
\hline $\begin{array}{l}\text { Velocidad del } \\
\text { procesamiento } \\
\text { (velocidad de de- } \\
\text { cisión y reacción) }\end{array}$ & $\begin{array}{l}\text { Habilidad de reacción ante estímulos sea para decidir o } \\
\text { responder. } \\
\text { Habilidad: tiempo de reacción simple, tiempo de reacción en } \\
\text { la decisión, velocidad de procesamiento semántico, tiempo de } \\
\text { inspección, velocidad de comparación mental (rapidez en la } \\
\text { comparación). }\end{array}$ \\
\hline
\end{tabular}




\begin{tabular}{|c|c|}
\hline Habilidad & Descripción \\
\hline $\begin{array}{c}\text { Inteligencia } \\
\text { lectiva y escrita }\end{array}$ & $\begin{array}{l}\text { Habilidad de crecimiento y profundidad de conocimientos y } \\
\text { destrezas de escritura y de lectura procesual y declarativa ad- } \\
\text { quirida por una persona. } \\
\text { Habilidad para escribir y leer discursos complejos (demuestra la } \\
\text { capacidad de comprender y habilidad de escribir una historia) } \\
\text { Habilidad: decodificación de la lectura, comprensión de la lec- } \\
\text { tura, comprensión lingüística verbal, habilidad de escritura, ha- } \\
\text { bilidad de usar la lengua madre, escribir y leer rápido. }\end{array}$ \\
\hline $\begin{array}{l}\text { Inteligencia } \\
\text { de conocimiento } \\
\text { cuantitativo }\end{array}$ & $\begin{array}{l}\text { Habilidad de crecimiento de profundización de los contenidos } \\
\text { adquiridos en torno al conocimiento numérico-cuantitativo. Lo } \\
\text { cuantitativo logra sintetizar algunas habilidades previas y trans- } \\
\text { forma lo experiencial en digital. Esto implica uso de la infor- } \\
\text { mación numérica, no razonamiento con este campo numeral. } \\
\text { Habilidad: matemático conocimiento, logro matemático. }\end{array}$ \\
\hline $\begin{array}{l}\text { Inteligencia de } \\
\text { conocimiento } \\
\text { general y } \\
\text { específico }\end{array}$ & $\begin{array}{l}\text { Habilidad de crecimiento, profundización y maestría en torno } \\
\text { a un dominio, una disciplina o materia especializada, que se di- } \\
\text { ferencia de la común dimensión cultural o experiencial de los } \\
\text { demás. Para el desarrollo ese conocimiento el sujeto se sometió } \\
\text { a mucha práctica y ejercicio sistemático, manteniendo un co- } \\
\text { nocimiento base. } \\
\text { Habilidad: conocimiento de diversas lenguas, lenguaje de señas, } \\
\text { destrezas de leer mirando el movimiento de los labios, informa- } \\
\text { ción general de la ciencia, conocimiento mecánico, contenido } \\
\text { conductual del conocimiento. }\end{array}$ \\
\hline $\begin{array}{l}\text { Inteligencia táctil } \\
\text { (habilidades } \\
\text { táctiles y de } \\
\text { caracterización } \\
\text { personal) }\end{array}$ & $\begin{array}{l}\text { Habilidad para desde las impresiones táctiles hacer juicios y leer } \\
\text { las sensaciones. Todo lo relacionado con juicios de estimulación } \\
\text { termal, estimulación espacial, juicios desde la piel, etc. } \\
\text { Habilidad: sensibilidad táctil. }\end{array}$ \\
\hline $\begin{array}{l}\text { Inteligencia } \\
\text { kinestética } \\
\text { (habilidades de } \\
\text { movimiento) }\end{array}$ & $\begin{array}{l}\text { Habilidad para leer el movimiento del propio cuerpo: posición, } \\
\text { peso, movimiento de músculos, tendones. De allí nace la capa- } \\
\text { cidad de controlar movimiento, gestos, capacidad de caminar, } \\
\text { expresiones faciales, gestos al hablar. } \\
\text { Habilidad: sensibilidad kinestésica. }\end{array}$ \\
\hline $\begin{array}{l}\text { Inteligencia } \\
\text { olfativa (habili- } \\
\text { dad olfativa) }\end{array}$ & $\begin{array}{l}\text { Habilidades que dependen del dominio de las sensaciones emo- } \\
\text { tivas que vienen por el sistema olfativo. } \\
\text { Habilidad: memoria olfativa, sensibilidad olfativa. }\end{array}$ \\
\hline $\begin{array}{l}\text { Inteligencia } \\
\text { psicomotora }\end{array}$ & $\begin{array}{l}\text { Habilidad para preformar movimientos motores del cuerpo } \\
\text { físico, con precisión, coordinación o fuerza: movimiento de de- } \\
\text { dos, manos piernas, etc. Esto implica un gobierno mental de } \\
\text { esos movimientos. }\end{array}$ \\
\hline
\end{tabular}




\begin{tabular}{|c|l|}
\hline Habilidad & \multicolumn{1}{c|}{ Descripción } \\
\hline & $\begin{array}{l}\text { Habilidad: fuerza estática, coordinación de múltiples miembros, } \\
\text { destreza manual y de los dedos, puntería, precisión de control, } \\
\text { equilibrio de cuerpo bruto. } \\
\text { Habilidad: }\end{array}$ \\
\hline $\begin{array}{c}\text { Inteligencia } \\
\text { de velocidad } \\
\text { psicomotora }\end{array}$ & $\begin{array}{l}\text { Habilidad para preformar rápida y fluidamente movimientos } \\
\text { motores del cuerpo físico (manos, pies, piernas), ampliamente } \\
\text { independientes del control cognitivos. } \\
\text { Habilidad: movimiento de velocidad de miembros, velocidad } \\
\text { para escribir, velocidad de articulación, etc. }\end{array}$ \\
\hline
\end{tabular}

Fuente: (McGrew, 2009; Carroll, 1993, 1997, 2005).

Elaboración: El Autor

El cuadro que precede ha sido construido sobre tres modelos de actividades cognitivas.

Habiendo tomado en cuenta la inteligencia no computacional, es comprensible que se asimile el análisis respectivo en torno a la experiencia y a la cognición.

\section{Experiencia-Cognición}

La experiencia no la tienen los sujetos, por ello para aprender primero hay que enseñarles a hacer experiencias. Esta se da por la vía sensorial o por el mundo límbico. La una para el externo y la segunda para el interno; ello no significa que no se intersectan las dos, que es propio del aprender, lo que implica que no hay modularidad fodoriana y que ya pone en crisis la perspectiva analítica del lenguaje y del conocimiento.

En el desarrollo de los infantes se constata que antes de elementos cognitivos como la lengua, primero se informa y condiciona los sentidos, sobre todo los del ojo y los de la audición, posteriormente se ejercitará la táctil. La intersección del enseñante es encontrar los niveles cognitivos lingüísticos con los de la sensorialidad o con los del sistema límbico.

El aprender sin experiencia suscita conocimientos especulativos e impertinentes, en cambio los conocimientos con experiencia son encarnados (Gelman, 1990), y pertinentes. Si la física se aprende sin pertinencia es improcedente, pero una vez que se la pone experiencia, surge la ley de la gravedad. 
Figura 1

Conjunción experiencia y cognición

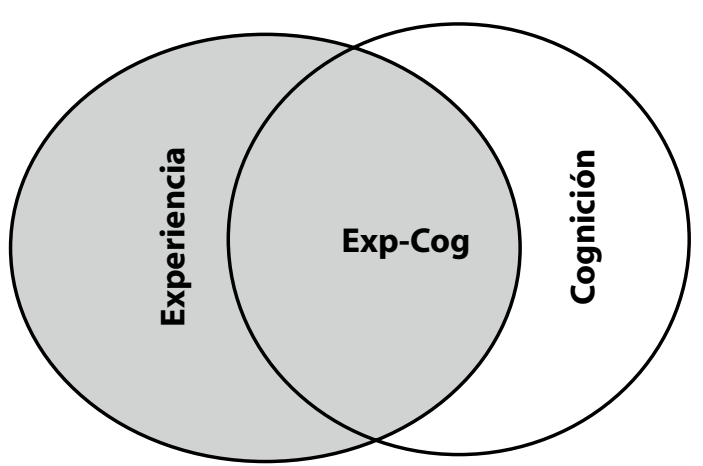

Fuente: La cognición con experiencia es pertinente, tanto experiencia interna y externa (San Martin, 2016)

Desde el punto de vista del desarrollo de los conocimientos, los niños primero aprenden las realidades de masa, de las cuales emergen principios de peso. Esto implica características propias de la masa, que son sensoriales y son puestas en serie; aquí es necesario la presencia de cosas. A partir de estos se hace construcción de conceptos. Entonces estos conceptos son más perceptuales-experienciales. Por las características de sustancia se abre al sentido de peso, tamaño, extensión (Gelman, 1990). Esta sustancia implica sucesión como en dominó creando la percepción de causalidad psicológica.

La segunda es a través de principios contables, que rigen para los números y por supuesto para las cantidades abstractas, en las cuales se mantienen de carácter más nominal, imaginados o substanciales, por ello son más abstractos y son aplicables a un amplio número de casos como representación, por ello son menos perceptuales, y por lo mismo no son tan aplicables a la memoria sensorial.

Ahora bien, el aprendizaje requiere elementos perceptuales, principios sustanciales en los cuales se entiende hasta el principio de causalidad, es decir de una forma más geométrica, para posteriormente ir a la forma aritmética. Si esto pasa con los números, otro tanto pasa con el lenguaje: sustancias y verbos con sus respectivas sustancias y representaciones abstractas. Cuando el niño entiende por experiencia podría asimilar mejor los elementos abstractos y contables. El aprendizaje tiene entonces este fundamento, de lo experiencial que se incluye en lo cognitivo, de lo cual se concluye que lo que lo experiencial debería encarnarse en lo cognitivo. 
Póngase el caso que se enseñe primero las cosas sin sustancia, que son las cuestiones contables y abstractas, entonces el aprender será insignificante, quedando insatisfecho el aprender y la cognición es sin experiencia, pues los contenidos abstractos están sin percepción; pero, si se tiene sustancia y no se hace el salto a los abstractos contables, igualmente queda en un empirismo solipsista que no se abre a los factores comunes del aprender.

Esto presupone comprende de forma más clara el punto sobre el cerebro relativo a la cognición lenguaje y cultura, por el nivel de complementariedad que exige este análisis con relación al planteamiento del artículo.

\section{Cerebro relativo a cognición social, lenguaje y cultura}

La relación entre las áreas cerebrales es más alta y definida entre los humanos. La comparación entre los humanos y los animales, da lugar a plantearnos el problema de la inteligencia relacionada con la vida social, con el lenguaje y por fin con la cultura. Hay una correlación entre la vida social y el lenguaje, pero también hay esa sin lenguaje cognitivo. Al mismo tiempo hay una relación entre vida social e inteligencia para pasar a una dimensión no solo de inteligencia social sino cultural (Tomasello, 1999; Hypothesis, 2010; Boyd, 2011, 2011a).

La cognición social está relacionada con una vida social, la cual está presente en diversas especies de animales. Los animales más sociales demuestran tener un cerebro más grande; ahora bien, esto es relativo, porque se ve que los pájaros, las abejas y los primates tiene son sociales, pero, sobre todo los dos primeros, no tienen un cerebro grande y diversificado, es decir tienen vida social, sin tener un gran cerebro. En estos hay vida social, pero no logran aprender, porque hay necesidad de un gran cerebro para aprender. Por lo tanto, no comporta que tener vida social abra la posibilidad a aprender. Para el aprender se requiere vida social y cerebro grande (Reader, 2003). La discapacidad cognitiva limita la respuesta a tratamientos psicosociales, de manera que hay una vinculación entre los procesos cognitivos y la vida social (Quee et al., 2014).

Pero, de cualquier modo, al menos para los primates, es imprescindible tener un cerebro grande. Éste consume mucha energía, mucho más que los demás organismos, y cuando están inmaduros más energía necesitan (Kuzawaa et al., 2014). En efecto un cerebro en desarrollo va lentamente y necesita tiempo, es decir se va conformando y podría hasta crecer descuidando otras dimensiones. El peligro, el ser presa de otros haría que un cerebro se dedique a correr y por tanto a no desarrollar facultades cognitivas y por lo mismo con un cerebro que no aprende. El humano no solo está sujeto a la supervivencia, sino a aprender; el humano no es presa. 
Un comportamiento preocupante, que perjudica el aprender y aumente la violencia y la supervivencia es el bullying que pone en peligro el desarrollo social del cerebro, porque el sujeto se hace presa de los demás. En un cerebro grande, que consume mucha energía, minerales y proteínas y un simple tejido, con la asamblea neural, se convierte en un núcleo para conocer y desarrollar destrezas no sería nada inteligente que retroceda y se dedique a estar sometido a la amenaza, a la defensa y hasta a la esclavitud. Un comportamiento de abuso retrocede la capacidad cognitiva del cerebro (Judith M. Burkart M. N., 2016). Inclusive hay que entender en esta dimensión el porqué del cuidado maternal por largo tiempo para que desarrolle la inteligencia. Esta es la base para aprender.

Una vida social implica aprendizaje social, imitación, seguimiento de la mirada y teoría de la mente (Fitch, 2009). Pero si a esto, que pueden tener otras especies, no se incluye el lenguaje, queda, pues, como vida social adaptable impresionante, pero con poca capacidad de aprender. Se acomodará socialmente, lo que en el darwinismo es adaptación a los ambientes, pero sin comunicación. Pero el humano además de esos comportamientos sociales, desarrolla una capacidad lingüística que expresa y se abre al entendimiento.

Así que el lenguaje es un plus, expresado y que abre al entendimiento, de la vida social por el cual se pasa de la imitación al aprender, a la mediación, a transformar la mente. Por el lenguaje el humano pasa no solo como social, sino que hace uso de ésta para desarrollar nuevas destrezas. Lo social como cognición que es propia de los humanos, la cual se hace presente en los humanos en las diversas etapas de su desarrolla, y que por el intercambio termina siendo no solo social sino un desarrollo cultural. La dimensión social se intercambia y por ella se desarrolla destrezas culturales (Tomasello, 1999).

Por lo tanto, lo social es para aprender, en el que el lenguaje es el intercambio social que se hace desarrollo cultural. Las capacidades sociales terminan siendo un mundo cultural.

Analizar el modelo humano para las máquinas de reconocimiento y de pensamiento, presuponen la importancia en torno a la temática planteada.

\section{El modelo humano para las máquinas de reconocimiento y de pensamiento}

La admiración que suscita las máquinas de inteligencia artificial reflejada en video juegos, reconocimiento de objetos y de rostros, traducciones lingüísticas, buscadores, etc. hace olvidar que esas son una imitación del 
cerebro humano, pero limitada. La codificación que sufre el cerebro, se traduce en elementos de cognición y de reforzamiento de ella, pero esto acto es similar al encauzamiento que sufre una vertiente de agua para la producción de la luz; en realidad no es que la energía eléctrica sea la causa, es pues el efecto, por ello una inferencia intencionada del agua; y una vez que ha movido las turbinas, el agua sigue su curso. Así mismo es la deducción del cerebro en instrumentos para el aprendizaje, son efectos cerebrales, por lo que el cerebro, después sigue su curso.

Así como del efecto se va a las causas, se va de los instrumentos creados para el aprender al núcleo que gestiona el aprender. La aplicación es una maravilla imitativa de la inteligencia natural, pero sólo de ella. Pues el ser humano aparte de inteligencia natural, tiene una escala, de sensibilidad, animosidad interior, y racionalidad lógica. Esto indica que el humano es mucho mejor en la originalidad del pensar, ciertamente biológicos; soluciona mejor ciertos problemas computacionales tales como sentido común, creatividad, aprendizaje de conceptos, adquisición y entendimiento del lenguaje, reconocimiento discursivo.

Habiendo analizado el aspecto no computacional de la inteligencia, con sus respectivas aclaraciones, se prosigue a comprender la deficiencia de la analítica en torno a la estructura cerebral, punto que tendrá en cuenta la crítica que desarrollo este artículo para inconsistencia de la dimensión analítica-empírica desde la conformación cerebral cognitiva.

\section{Deficiencia de la analítica en torno a la estructura cerebral}

El empirismo se ha dedicado a la filosofía analítica y se ha convertido en el paradigma para el análisis de la lengua. De entre todas las formas del empirismo la más analítica es la del empirismo del siglo XX que se asoció a las formas de psicología del behaviorismo. Así pues, si el psicólogo conductista analiza el aprender y la estructura de la personalidad desde los comportamientos de los sujetos, es decir desde los hechos visibles, por lo que infiere que el hombre es la suma de sus actos. Este mismo criterio para la psicología, estuvo precedido del análisis de la lengua, en el que los productos lingüísticos remiten al ingreso sensorial, el cual podía ser hasta medido estadísticamente, así que análisis de la lengua no es sino una descomposición de los términos y de las proposiciones para buscar un referente o un hecho que le garantice su naturaleza. Además, como la lengua es la base para la lógica (San Martín, 2012, 2016), ésta está en el 
deber de fundarse y justificarse, igualmente en los hechos, su naturaleza lógica, es decir allí se justifica y la valida.

Pero, aunque se ha convertido el empirismo en el paradigma para la filosofía analítica, según la estructura cerebral otro es itinerario de la formación lingüística; en efecto desde la formación y funcionamiento cerebral, la lengua se remite a un código interior a la especie humana que se articula con los datos externos.

Por lo tanto, entrará en crisis el paradigma tradicional de la filosofía analítica, que tiene criterio de validez con lo antes analizado, pudiendo en el siguiente punto relacionar la experiencia y el lenguaje.

\section{La relación entre experiencia y lenguaje}

En la filosofía en general se asume una versión naturalista para la materia; ésta se convierte en la base para toda posterior construcción del conocimiento, por lo que el lenguaje se insertará en esos cánones. El lenguaje es el que tiene que remitirse a la materia para un enunciado cualquiera. Este efecto crea hasta una dependencia psicológica, dado que la materia se convierte el punto de objetividad, por tanto, en el centro donde se puede tener más certeza; en cambio lo no material y por tanto, lo lingüístico sugiere un pantano complicado que en la parte más conflictiva asume roles de paranoia; en efecto, todo el proceso del conocer debe remitirse a la materia, por ello los sentidos, la emociones, la inteligencia y la misma lógica.

En fin, parece que triunfa una perspectiva parmenidiana y aristotélica. Esta hasta se ve en las tendencias empiristas modernas, que terminan con el empirismo inglés y la filosofía analítica y el círculo de Viena. Ayer por ejemplo asume que un enunciado es literalmente significativo si equivale a analítico o empíricamente verificable (Ayer, 1965, p. 9). El significado depende de ser analítico o empírico.

En cuando analítico, comporta que los enunciados sintéticos no son significativos, puesto que están asociando aspectos que quizás no le correspondan. Lo que es analítico es producto de la razón, por ello son los a priori, y no aumentan en nada el conocimiento, antes bien sólo reflejan las realidades de modo más explicado. Ello es una anulación de los conocimientos científicos, que refieren no analíticos sino relaciones de conjunción, separación, implicación, es decir que hay una lógica que gobernará el conocer, por ello los enunciados compuestos no son literalmente significativos. Lo que es analítico son las pruebas espejo: lo que está al frente eso se refleja. 
Por otro lado, los enunciados empíricos serán significativos en cuanto son verificables sensorialmente, con la posibilidad de ser refutados por la actualización de la experimentación, por ello el mundo empírico jamás es tribunal definitivo de significatividad, en cuanto que la posibilidad de refutar queda abierta; no es posible establecer dogmas desde el empirismo, sin embargo, puede tornarse dogmático cuando asume que, si es verificable, sólo allí es significativo. Aquí los eventos lingüísticos, no pueden referir nada de sí, puesto que no tienen relación directa con la experiencia; ellos solo dirán lo que se verifica. La lengua nace aquí como descripción de los eventos físicos.

\section{Lengua y materia, deben ser sensoriales}

El fundamento de la lengua es la materia. Las proposiciones empíricas no son añadidas, en nada, por la dimensión lógica. No es el argumento el que verifica, sino el dato que está en la base. Lo que valida es el dato sensorial.

En la aplicación que se haga de este principio, es por fin infiel al mismo Newton, para el cual después de los datos hay la abstracción de un factor común, que él lo llama principio, el cual no es una cosa verificable sensorialmente, sino desde los datos de la lógica; ese principio es una desmaterialización de los datos sensoriales y la plasmación en una memoria lógica, desde la cual se aplica a otras realidades, inclusive a eventos no contemplados en ese campo inicial de datos. De aquí nacen las excepciones, que es un dato que no quepa dentro del principio, pero ante todo es contradictorio con los datos que se experimentó. Esto es lo que se llama un pato feo, un cisne, que no calza en el común de los patos, para la cual hay que hacer otra teoría. En fin, la línea de verificación es la experiencia.

Pero preguntémonos ahora, ¿cuál es el problema que está subyacente en este caso al empirismo, pero que también, de modo inverso a ese, en el racionalismo? El problema es el desconocimiento de la estructura cerebral que regula el aprendizaje y el conocer. El cerebro no es ni naturalista ni convencionalista, por ello tampoco empirista ni racionalista. La arquitectura de éste es: tiene áreas que son exclusivamente sensoriales y otras emocionaleslingüísticas. Las columnas para el conocimiento son el área sensorial y el límbico. La viga que junta esas dos realidades es la que produce el conocer.

En el cerebro una es el área sensorial y otra la lingüística. Las dos son distintas estructuras que están en el cerebro y tienen diverso origen: Los sentidos están remitidos al exterior; la lengua al interior, más relacionada con el sistema límbico (San Martín, 2012, 2016). 
A nivel de desarrollo del sujeto primero está la marca sensorial; ésta está anticipada en los seres incluidos los no mamíferos; conocen la realidad externa, reciben los estímulos para sus órganos sin mandarlos al interior, que no la tienen. Es en efecto en estos seres la máxima potenciación de la conducta en proporción al estímulo recibido; no hay aumento desde el ser que lo recibe. Los mamíferos inferiores, incluidos los marsupiales, en cuanto generados en el interior placentario tienen sistema límbico, por lo tanto, pueden registrar en su memoria los datos que han recibido. Esto pasa igualmente con los mamíferos superiores, tales como el chimpancé y el orangután, que tienen un largo periodo de fecundación en el útero, pero están anegados en su interioridad y, por fin hasta conformados, en cuanto que no tienen la dimensión lingüística, que es capaz de expresar lo que pasa interiormente (San Martín, 2016). Tienen emociones, pero no sistema lingüístico.

Así la lengua está sostenida no por el exterior, sino como eco emocional, como eco de la vida. Es más, una facultad de formalización de la emoción, de las profundidades inexpresables del ser humano, que de contenidos mismos. La emoción sin lengua es un material amorfo y caótico; es similar a tener combustible fuera de un contenedor, lo que lo hace peligroso; las emociones son puro movimiento, llenos estados de placer e insatisfacción, de necesidad, de dolor. Son estados emocionales indecibles; por ello son experiencias duras que quedan sin forma, pero que lastiman a la persona. La emoción negativa es la herida que tiene el sujeto y que no puede expresarla.

En este campo aparece el grito, que la emoción en donde se articula en voz, y se hace más o menos semántica. La voz nace de las entrañas del ser humano, es como dar a luz a las emociones indecibles; por ello es la liberación del sistema límbico. En este sentido la palabra es la versión psicológica del ansia, que da a luz lo que gesta las vísceras interiores. El silencio de la evolución, de la continua relación entre herencia y ambiente termina siendo fonético. Junto a la herencia y al ambiente aparece la le lenguaje que manifiesta.

La palabra nace por tanto naturalmente del sistema límbico, como las especies nacen del útero. Es la epifanía de las emociones. No nace la palabra como productos de los estados sensoriales, esto no necesitan la palabra. Estos se bastan a sí mismos para decir lo que acontece. El exterior no grita, sino sólo estimula y el sujeto responde sintiendo, en cambio el interior produce algo nuevo de sí, la voz, es el grito, como el previo de la palabra.

El lenguaje no es una estructura exclusiva para el conocimiento; esa está relacionada con la emoción. La separación lenguaje y emoción, 
y por lo mismo la separación del conocimiento de la emoción está dada por la procesual paralelización del lenguaje y emoción. Estas estructuras en sus orígenes no están separadas; la emoción y el lenguaje, en sentido de voz y movimiento, están íntimamente relacionados; quizás por eso que no se desató el lenguaje de manera hablada y por fin conceptualizada. Voz y emoción van a la par (San Martín, 2015). El lenguaje, en el sentido de voz, hay que ir a encontrarlo en el sistema límbico, pues allí está el centro de las emociones (Deacon, 1989; Lieberman, 2000; Mithen, 2007).

Por el lado sensorial, sea la visual, la auditiva, la táctil no necesitan de la lengua. El cerebro tiene, esas, separadas de la expresión. Lo visual no se hace más tal si es dicho y lo mismo en relación a los otros sentidos, porque esta capacidad es en relación directa con la materia, así que sin lenguaje pueden ser asimilados. El componente significativo de áreas sensoriales es que haya estímulo externo y un órgano que recepta. Todos saben que es visual sin decir o auditivo sin manifestar. La naturaleza de estos no es la lengua. Véanse a los animales, tienen áreas sensoriales y saben manejarse, sin palabras, porque los sentidos son, por así decirlos, "analíticos mudos", en cuanto que recibe el estímulo y siente el órgano en cuestión, su naturaleza es sentir ese preciso estímulo; no se puede saber que es amargo porque lo dice verbalmente, sino porque lo siente la lengua; son analíticos porque son asimilados por los sentidos.

Los sentidos, cuando hablan, están asumiendo una pseudo naturaleza; algo que no les corresponde. El lenguaje para ellos es un sobrepuesto a su naturaleza, que podría hasta destruir su analítica muda, puesto que puede terminar desvirtuando su ser. El cerebro se arregla bien con los sentidos y mucho tiempo no ha necesitado que le digan. Los chimpancés se arreglan bien con los sentidos y no porque se les enseñe a hablar logran variar la ontología sensorial. Por lo tanto, las áreas sensoriales son mudas por naturaleza.

Ahora bien, no se puede negar de la originalidad de la lengua, que sea deudora del sistema límbico y que poca cuenta debe rendir a las áreas sensoriales. La lengua no es precisamente para servir al sensorial, sin embargo, un uso inadecuado de la lengua podría dar lugar a una excesiva expresión de la sensorialidad o a enmudecer lo emotivo.

Por el lado de la expresión de la sensorialidad. Ésta por naturaleza da a la lengua una naturaleza subsidiaria; no tiene una importancia decisiva en el conocimiento, sino como comunicador de los eventos del ser; en efecto la lengua dice lo que acontece en el ser, sin añadir ni quitar nada de la sustancia. Además, ciertamente de modo negativo, es oportunista, pues asumiendo que lo que es material y sensorial no puede expresarse 
de modo claro; acude al lenguaje como el vocero de los actos de la experiencia sensorial; el lenguaje se convierte en la voz de la sensorialidad; el contenido son los datos de las áreas cerebrales. Pero también, la sensorialidad debe sacrificar parte de su naturaleza, en cuanto, que ésta es muda y no por imposición sino por naturaleza, por lo que se sobrepone una realidad que no le corresponde. Entonces surge en medio de la referencia de la lengua, hacer lo que es material en voz; descifra lo corporal en fonético.

Dos naturalezas diversas tienen que conciliarse. No hay un reflejo de la lengua con respecto a la materia, por sí mismo, sino una traducción y una descifración. Aquello que es material se traducirá en voz, será una conversión de lo material en voz; pero las traducciones no asimilan la naturaleza interna total de la realidad traducida, puesto que es un superpuesto, por lo tanto hay dos posibilidades: 1 . El ser, lo material, lo sensorial no será dicho plenamente por la lengua, sino sólo aproximativamente, es decir no será un reflejo de su naturaleza; de aquí que la lengua será una versión digital de la corporal, por lo que las teorías que nacen de la estructura material y sensorial no serán verdaderas, sino aproximativamente tales. Por otro lado, 2. La lengua aún en el caso de ser fiel a la materia a traducir, debe descifrar ese contenido material, por lo que aún en su presunta fidelidad; interpreta; por ello eventualmente dirá más de lo que la misma materia sostiene o también menos. El lenguaje, que tiene su naturaleza, insertará una dimensión toda suya a lo que está a la base; en este sentido el lenguaje no es traductor, sino también dador de la naturaleza a lo que está diciendo, por lo que amplía el contenido traducido.

Así los contenidos de lo sensorial serán descifrados por la lengua, que presentará en proposiciones o enunciados.

Pero también, la sensorialidad dicha puede afectar a la naturaleza del lenguaje, que está para decir naturalmente lo interior. Cuando se percata que puede decir de la naturaleza externa, descuida su especialidad interna y se vuelca a lo que presuntamente es más objetivo. Se acomoda y se sacrifica al externo, a lo material. Se especializa en res externa, pero descuidará su naturaleza de cognición. La dedicación al exterior dio lugar al nacimiento de una física del lenguaje y por lo tanto al desarrollo de las ciencias pertenecientes a ese nivel, para los que fue posible hasta hacer modelos que figuran el funcionamiento de la naturaleza externa.

La matematización de la realidad débase a una enumeración de los casos, haciendo que el caso empírico esté sostenido por el lenguaje numérico. 
El momento de la relativización del externo y el inicio de la preocupación del dictado de la interioridad está dado cuando al referente se lo toma como "el fenómeno", es decir que la realidad no es accesible directamente, sino a través de los mismos: los fenómenos. Estos son la manifestación de la realidad, que no viene directamente de los sentidos que no pueden hacer ninguna interpretación, sino de la pregunta que la interioridad es influye en el externo y pone en duda la presentación y es tomada como fenómeno de las cosas.

Así se logra comprender la realidad misma de la inconsistencia de la realidad analítico- empírica, aunque resulta relevante aclarar los enunciados analíticos como ilusión empírica, para que por medio de estos se atestigüe la inconsistencia.

\section{Los enunciados analíticos como ilusión empírica}

Los enunciados en Carnap son verificados a través de la experiencia, sostenida en los órganos sensoriales. Esta verificación es directa e indirecta (Carnap, 1965, p. 22), la primera es la confrontación directa con los sentidos; la segunda es una conjunción de elementos separados que se unen por virtud natural, es decir que están separados pero se encuentran en la solución de un problema: así por ejemplo la atracción de los metales al imán, esto es propio de los metales, pero se confirma en la acción visible y palpable de atraerse (Carnap, 1963).

Sin duda alguna, la sensibilidad de Carnap, aunque esté supeditado a la verificación por la sensibilidad, en el hecho mismo de la ciencia, termina no aumentando el conocer, dado que para éste se necesita una estructura lógica que recepte las constantes de las realidades; es decir que el aumento del conocer no viene solo por los datos dados por los sentidos, pues aquello sería solo un archivo de datos, sino que viene de los datos articulados por categorías, por ello se orientan a la compresión en vista de la combinación lógica mental, antes que a la sensorial.

En la misma verificación indirecta, aunque aparentemente esté aumentando el conocimiento con los datos, sin embargo, está en la misma línea de los conceptos analíticos. Así pues, la naturaleza de los magnetos es atraer los metales, por lo que es interno a su mismo ser, su predicado está incluido en su misma naturaleza, así que el encuentro de los metales no es sino el ejercicio de su naturaleza, por lo que no es extraño ni ninguna síntesis, sino aplicación de su naturaleza. Así como se verifica indirectamente, del mismo modo está ejerciendo su mismo predicado. 
Los conceptos analíticos son los que no aumentan el conocimiento, su predicado está incluido en su sujeto. Tal naturaleza no implica que esté en su misma estructura corporal, sino en la relación con los otros, así que el imán en su mismo sujeto implica la atracción de los metales, por lo que éstos están incluidos en su ser y, a su vez los metales afines a él, tienen en su naturaleza este ser atraídos, por ello son juicios analíticos en sí mismo. Así como el agua moja la tierra, pues su virtud es mojar, otro tanto el imán atrae los metales, los dos casos son analíticos.

Los enunciados empíricos, cuando son descritos o narrados, son analíticos. Esta narración no comporta ninguna comprensión, ni reflexión, sino asociación de las áreas vecinas, esto es la del POT, las cuales son prestadas fonéticamente por el lenguaje. Una cosa es decir comprensivamente, pero otra es decir de lo que acontece en los sentidos. La asociación de los sentidos expresado verbalmente da lugar al área de Brocca que dice solo de los elementos sensoriales, sin añadir nada de lógica ni de significado.

Por lo tanto, no hay aumento del conocimiento, sino verificación sensorial.

Por otro lado, los ideacionalistas son los filósofos empiristas, tales como Hobbes, Locke, Hume, Frege, que dan a las palabras un sentido diferente al que le dan los referencialistas.

Por ello no son referencialistas en sí mismo, sino que trabajan con la representación de la cosa en la idea (Locke, 1986, p. 394).Así que hay una estructura cognitiva que es capaz de receptar la información exterior, a través de los sentidos, a la que se le da una característica nueva con la voz; así para éstos hay ya sensaciones y percepciones, como también el concurso de la memoria. El significado está relacionado con la palabra

La palabra está en el centro, entre la idea y realidad, según los ideacionalistas.

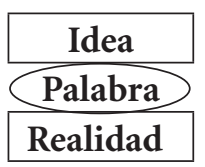

Entonces la palabra es la representación de la cosa.

La crítica que se infiere a la filosofía analítica, tanto en el lenguaje como en las proposiciones, es que la defensa de la pureza de la naturaleza analítica es insostenible, puesto que no resiste la estructuración del lenguaje. Esta se remite a una condición de la especie humana que articula los datos que la exterioridad presenta. 
Si el lenguaje fuese solo un estado que refleja lo exterior, al modo de reflexividad, sería descomplicado hacer el análisis, dado que una totalidad externa se ha hecho fonética y, así como se separa las sílabas de las palabras, del mismo modo se separan las cosas del contenido lingüístico, o también, así como de un rompecabezas se separa las piezas que lo componen, de modo similar se separarían las cosas de las palabras. Pero como el lenguaje tiene esta estructura esencial-cerebral, no es posible llegar a separar la estructura última del lenguaje, puesto que allí están insertados elementos vitales, en cuanto que está presente el sistema límbico.

Por lo tanto, el estatuto de la analiticidad empírica es insubsistente.

\section{Conclusiones}

En cuanto a la naturaleza de la cognición: La cognición es un proceso de vertebración de los elementos del externo, aún los digitales, con los propios de la especie. La inteligencia, en efecto, se va agarrando y enredando siempre que haya una base en donde aferrarse. La anulación de la base en donde enredarse, hace que todo permanezca en un conocimiento rastrero, expresado en instintos o en el dominio de los sentidos.

La cognición es una construcción enredada, por ello no es de sujetos solitarios, es decir que la cognición es social y cultural, por la cual se insertan elementos, no solo de la exterioridad, aún digital, sino de la profundidad y necesidad biológica y trascendental humana.

En cuanto a la facultad de la inteligencia: Es una facultad capaz de abarcar diversidad de eventos, para intencionarlos a habilidades altísimas, tales como el razonamiento lógico, insertando cuidadosamente los elementos sensoriales y límbicos.

Tiene la habilidad de separar los elementos provenientes del exterior, aún digitales, y de ponerlos bajo una sintaxis adecuada, y de perturbar su misma naturaleza, proyectando que la sintaxis viene del exterior y no de la capacidad categorial propia de la inteligencia. Producto de esto nació todo el contundente contenido, y cientificidad de la ciencia física, afincada desde el exterior, pero que posteriormente se absolutizó, dejando la idea que el que arma todos los contenidos mentales es únicamente el exterior.

La inteligencia sabe articular los elementos externos en una sintaxis interna (límbica-lingüística), desde la cual nace la semántica. La sintaxis no la pone solo la realidad externa, sino también la estructura categorial de la inteligencia, desde la cual se forma la semántica. 
En cuanto a la analítica: Es posible ser analítico, pero no en el sentido atómico de las ciencias físicas, sino sustentado en un fondo de cerebro inteligente, es decir se es analítico, porque hay una base cerebral. Si no hubiese este andamio, no hay ningún género de conceptos y proposiciones.

El cerebro sí es analítico, pero exclusivamente con los datos que vienen por las áreas sensoriales, puesto que éstas receptan y son capaces de desintegrar lo que receptan. Por otro lado, si el cerebro fuese solo sensorial, sin duda que sería sólo analítico y conjuntivo rudo, pero la sensorialidad entra en sincronía con las otras áreas cerebrales, con lo que termina la dimensión computacional de las cosas e inicia la biología del conocer, cuya característica singular es generar.

El empirismo clásico, ideacionalista, y el positivismo lógico moderno y contemporáneo, más referencialista, sucumbe ante la estructura dinámica del cerebro, que es antes que nada sintáctico-semántico, es analítico-vital y sintético-vital. Por ello esos empirismos son insubsistentes.

\section{Bibliografía}

AYER, Alfred

1965 Lenguaje, verdad y lógica. México. Fondo de Cultura Económica. BOYD, Robert

2011 Rapid cultural adaptation can facilitate the evolution of large-scale cooperation. Behavioral Ecology and Sociobiology, 65(3), 431-444.

2011a The cultural niche: Why social learning is essential for human adaptation. Proceedings of the National Academy of Sciences of the United States of America. 108, 10918-10925.

BURKART, Judith M. et al.

2016 The evolution of general intelligence. (C. U. Press, Ed.) To be published in Behavioral and Brain Sciences, 1-63.

\section{CARNAP, Rudolf}

1963 Filosofía y sintaxis lógica. México : UNAM.

1965 La superación de la Metafísica En: El Positivismo Lógico. México: Fondo de Cultura Económica.

CARROLL, Jhon

1993 Human cognitive abilities. En: A survey of factor-analytic studies. New York: Cambridge University Press.

1997 The three-strtum theory of cognitive abilities. En: D.P. Flanagan \&. P.L. Harrison, Contemporary intellectual assessment: Theories, test and issues (pp. 122- 130). New York: Guilford.

2005 The three-stratum teory of cognitive abilities. En: D.P. En C. i. Flangan \& P.L. Harrison (Eds), Theories, test and issues, 2nd Ed. (pp. 69-76). New York: Guilford. 
DEACON, Terrence

1989 The neural circuitry underlying primate calls and human language. Human Evolution, 4(5), 367-401.

FITCH, Tecumseh

2009 Biology of Music: Another One Bites the Dust. Current Biology, R403-R404.

GELMAN, Rochel

1990 First principles organize attention to and learning about relevant date: Number and the animate/inanimate distinction as examples. Cognitive Science 14, 79-106.

GOTTFREDSON, Linda

1997 Why g matters: The complexity of everyday life. Intelligence, 79-132.

TOMASELLO, Michael et al.

2010 The Cultural Intelligence Hypothesis.Research Articles, http://www.sciencemag.org/.

KUZAWAA,Christopher. et al.

2014 Metabolic costs and evolutionary implications of human brain development. PNAS, 36.

LIEBERMAN, Matthew

2000 Intuition: A Social Cognitive Neuroscience Approach. Harvard University.

LOCKE, Jhon

1986 Ensayo sobre el entendimiento humano (Trad. de E. O'Gorman ed.). México: FCE.

MCGREW, Kevin

2009 CHC theory and the human cognitive abilities project: Standing on the shoulders of the giants of psychometric intelligence research. Intelligence. Elsevier.

MITHEN, Steven

2007 Why are some handaxes symmetrical? Testing the influence of handaxe morphology on butchery effectiveness. Journal of Archaeological Science, 883-893.

OLSSON, Sven

2016 The unconscious zone: The secret life of your brain. 2016. Haftad

QUEE, Piotr. et al.

2014 Insight change in psychosis: relationship with neurocognition, social cognition, clinical symptoms and phase of illness. Acta Psychiatr Scand. 129, 126-133.

PINKER, Steven

2010 The cognitive niche: Coevolution of intelligence, sociality, and language. PNAS, 8993-8999.

READER, Simon

2003 Innovation and social learning: individual variation and brain evolution. Biology - Research Article.

SAN MARTín, Rómulo

2012 El pensamiento incorporado percepcional-lingüístico-lógico/the embodied, perceptional, linguistic and logic thougth. Sophia 13/ Colección de Filosofía de la Educación.

2015 La cognición incorporada: el contenido y la justificación del enfoque perceptocomprensivo del conocimiento. Tesis Doctoral. Salamanca. 
2016a El lenguaje incorporado desde y para la cognición incorporada. Sophia 20/ Colección de Filosofía de la Educación.

2016b Inconsistencia de la dimensión Analítica-Empírica desde la conformación cerebral cognitiva. Sophia 21/ Colección de Filosofía de la Educación.

SEMENDEFERI, Katerina. et al.

1998 Limbic Frontal Cortex in Hominoids: A Comparative Study of Area 13. American Jounal Of Physical Anthropology, 129-155.

TOMASELLO, Michael

1999 The Cultural Origins of Human Cognition. Harvard University Press.

TOMASELLO, Michael., \& CALL, Josep

1997 Primate cognition. New York: Oxford University Press.

TURING, Alan

1950 Computing machinery and intelligence. Mind, New Series, Vol. 59, No. 236

Fecha de recepción del documento: 15 de agosto de 2016

Fecha de revisión del documento: 15 de septiembre de 2016 Fecha de aceptación del documento: 20 de noviembre de 2016 Fecha de publicación del documento: Enero de 2017 